\title{
Wake Identification Based Wake Impact Alleviation Control
}

\author{
Jana Ehlers ${ }^{*}$, Dietrich Fischenberg ${ }^{\dagger}$ and Dominik Niedermeier ${ }^{\ddagger}$ \\ German Aerospace Center DLR, Institute of Flight Systems, Department Flight Dynamics and Simulation \\ Lilienthalplatz 7, 38108 Braunschweig, Germany
}

During a wake vortex encounter an aircraft is exposed to strong unsteady disturbance velocities which can lead to significant changes in the aircraft attitude and flight path. This can represent a severe safety risk and can result in injuries to the passengers and crew as well as damages to the aircraft. The application of a wake impact alleviation control system can considerably decrease the aircraft's response during the wake encounter, and hence diminish the pilot workload while reducing the potential risk to the passengers, crew, and aircraft. The realization of a wake impact alleviation controller presented here is based on a forward-looking LiDAR sensor. The information about the disturbance velocities in front of the aircraft is used to determine the control surface deflections that compensate the wakeinduced disturbance moments. The novel approach of this concept is the combination of the wake impact alleviation system with a wake identification algorithm. Due to the integration of the identification algorithm it is possible to apply the wake impact alleviation with LiDAR sensors restricted to line-of-sight measurements only. In this case the LiDAR sensor only detects the flow velocity in the direction of the measurement beam. A measurement of the full velocity vector of the flow field upstream of the aircraft, which LiDAR sensors of the foreseeable future will most likely be unable to provide, is not necessary for this approach. The wake identification based wake impact alleviation is assessed for different encounter scenarios and a brief sensitivity study is performed for the most important parameters of the wake identification. It is shown that the wake impact alleviation control system significantly reduces the wake-induced attitude change of the aircraft and thus represents a promising concept to increase safety during wake vortex encounters.

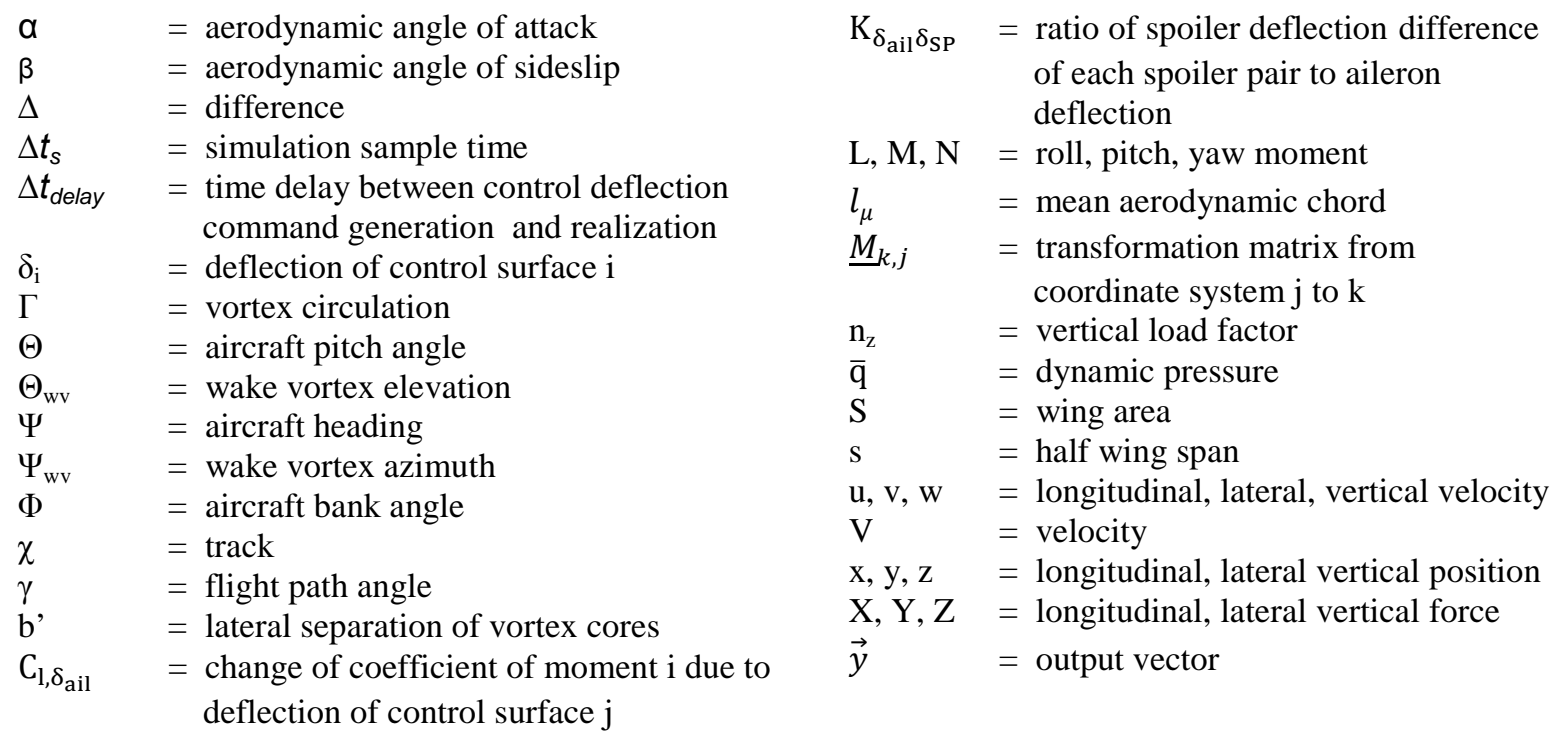

\footnotetext{
${ }^{*}$ Research scientist, German Aerospace Center, jana.ehlers@dlr.de

${ }^{\dagger}$ Research scientist, German Aerospace Center, dietrich.fischenberg@dlr.de

† Research group leader, German Aerospace Center, dominik.niedermeier@dlr.de
}

1 


\begin{tabular}{ll}
\multicolumn{2}{l}{ Abbreviations } \\
a/c & aircraft \\
ADIRU & Air Data Inertial Reference Unit \\
ADS-B & $\begin{array}{l}\text { Automatic Dependent Surveillance - } \\
\text { Broadcast }\end{array}$ \\
AIM & $\begin{array}{l}\text { Aerodynamic Interaction Model } \\
\text { CG }\end{array}$ \\
Center of Gravity \\
GPS & Global Positioning System \\
LES & Large Eddy Simulation \\
LiDAR & Light Detection And Ranging \\
LoS & Line of Sight \\
NED & North East Down \\
OWI & Online Wake Identification \\
WIAC & Wake Impact Alleviation Control
\end{tabular}

\begin{tabular}{ll}
\multicolumn{2}{l}{ Subscripts } \\
A & aerodynamic coordinate system \\
ail & aileron \\
b & body-fixed coordinate system \\
comp & computed \\
E & east \\
elev & elevator \\
L & left-hand side \\
N & north \\
meas & measured \\
pred & predicted \\
R & right-hand side \\
rud & rudder \\
SP & spoiler \\
TAS & true airspeed \\
WV & wake vortex
\end{tabular}

\section{Introduction}

Wake vortices represent a serious safety hazard for aircraft traffic. They are the undesired side product of the lift generated by an aircraft. For a following aircraft flying into the wake vortex of another aircraft the wake vortex can cause dangerous aircraft reactions such as a sudden rapid roll motion. This poses an increased workload on the pilot and may lead to injuries of the passengers and crew, and structural damages to the aircraft. In extreme cases a wake vortex encounter can even provoke an aircraft crash. ${ }^{1,2}$

An approach to reduce the impact of a wake vortex encounter and thus decrease the risk of hazardous aircraft reactions is the application of a wake impact alleviation control function. The German Aerospace Center (DLR) developed a wake impact alleviation controller (WIAC). It is applied in combination with a forward-looking light detection and ranging (LiDAR) sensor and uses the information about the flow field ahead of the aircraft to determine control commands that compensate for the forthcoming wake-induced disturbance. This concept has been assessed in different studies before. Ref. 2 to 5 demonstrate the successful application of the concept for a forwardlooking measurement sensor that provides the full vector of the wake vortex wind velocities in front of the aircraft. At Technische Universität Berlin the disturbance rejection concept is studied as well and applied in a modified approach. ${ }^{6}$ The disturbance affecting the aircraft is considered over a certain period of time into the future and the control surface deflections are commanded that lead to an optimal alleviation of the wake disturbance over this time interval. In this application the wake impact alleviation can, however, only be used without a pilot in the loop because the aircraft reaction cannot be predicted over the future time interval if the pilot commands influence the aircraft behavior. The approach taken in this paper is to design a wake impact alleviation controller which can be used as a pilot assistance system and reduces the workload of the pilot during the encounter. The application is thus focused on the current disturbance and does not aim to find an optimal reduction of the aircraft response over a period of time as realized in Ref. 6.

The main advantages of the concept of wake impact alleviation as disturbance rejection with a forward-looking sensor are its fast response time and its independence of the basic flight control system of the aircraft. The fast response time is possible due to the forward-looking measurement and the feedforward architecture. This avoids the negative influences of sensor time delays and provides the possibility to compensate for actuator delays. As the wake impact alleviation control system only determines additional control surface commands that are added to the control surface deflection commanded by the basic controller, this concept is applicable for all manual and automatic flight modes without any changes or adaptions of the basic control system. This allows a wide and flexible range of application of the system. Moreover it could be installed subsequently on existing aircraft configurations without the necessity of redesigning the present flight control laws. This is an advantage compared to other approaches of wake impact alleviation systems which develop a completely new feedback control system for the aircraft and considered a wake vortex as one of the disturbances the control system should be able to cope with. ${ }^{7,8}$

To date regular commercial aircraft are not equipped with LiDAR sensors. The capabilities of future sensors are unknown and thus not clearly specified at present. Previous applications of the disturbance rejection on the basis of forward-looking measurement concepts assumed that the full vector of the disturbance wind velocity would be 
known. ${ }^{3-6}$ Current developments of the sensor technology suggest, however, that sensors for the detection of the full velocity vector will not be realizable with practicable effort in the near future. Feasible LiDAR sensors will most likely only be able to measure the velocity in the direction of the measurement beam, the so-called line-of-sight (LoS) velocity. For wake vortex encounters with small encounter angles this means that most of the information about the disturbance velocity is lost because the main velocity components occur perpendicular to the flight direction. The direct use of the line-of-sight measurement of the LiDAR sensor is thus not feasible for a successful wake impact alleviation. A solution of this problem is the integration of online wake identification into the wake impact alleviation control system. The wake identification algorithm uses the line-of-sight measurement provided by the LiDAR sensor at different measurement points ahead of the aircraft to identify the characteristic parameters of the wake vortex. The wake impact alleviation control system derives the wake-induced disturbance from the identified parameters and computes the control surface deflections to compensate this disturbance.

This paper presents the implementation of the wake impact alleviation controller and assesses its application under different conditions. Section II gives a detailed description of the alleviation control system and an overview over the wake identification algorithm. Section III analyzes the success of the wake impact alleviation under idealized conditions. It is assumed here that the wake vortex parameters, on the basis of which the control commands for the disturbance rejection are determined, are perfectly known. Afterwards the attainable alleviation for the combina-

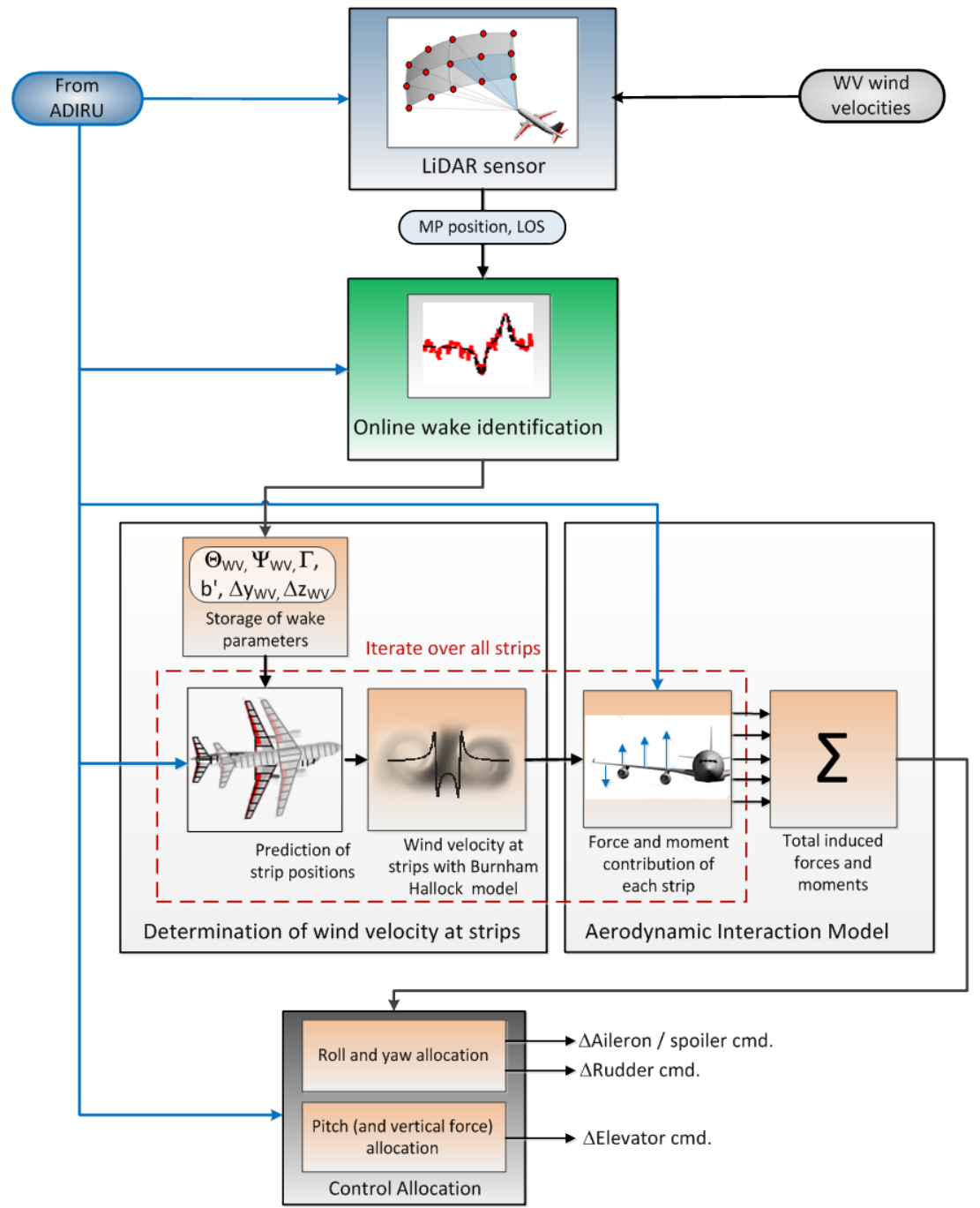

Figure 1. Workflow of online wake identification and wake impact alleviation. tion of the wake identification and the wake impact alleviation control system is analyzed for different encounter scenarios and sensor characteristics in Section IV. For a specific encounter scenario the sensitivity of the success of the wake impact alleviation with respect to the quality of the identified parameters is assessed in Section V. Section VI concludes the paper with a summary and outlines future work.

\section{Concept of the Wake Impact Alleviation Control System}

The wake impact alleviation control system presented here uses the information on the wake vortex flow field of a forward-looking sensor to derive the control surface deflections that compensate for the wake-induced disturbance. An overview of the principle of the wake impact alleviation system is given in Fig. 1. The forward-looking sensor measures the flow field in a short range ahead of the aircraft. This information is used to derive a wake vortex model 
and calculate the wake-induced disturbance on the aircraft. By inverting the aerodynamic model of the aircraft the control surface deflections that are needed are determined to compensate for the wake-induced aircraft response.

In detail this approach is realized as follows: For the forward-looking sensor a so-called Doppler LiDAR (Light Detection And Ranging) sensor is used. This sensor permits to measure the wind component along a laser beam using the frequency shift of the backscattered signal. Current developments indicate that these sensors will only be able to detect the flow velocity in the direction of the measurement beam in the next few years. For the most critical

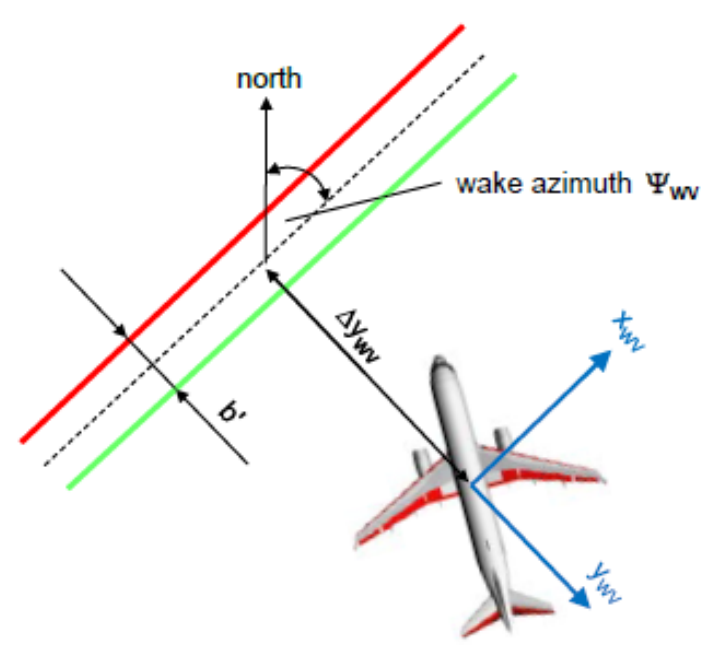

Figure 2. Geometry of LiDAR output parameters (top view).

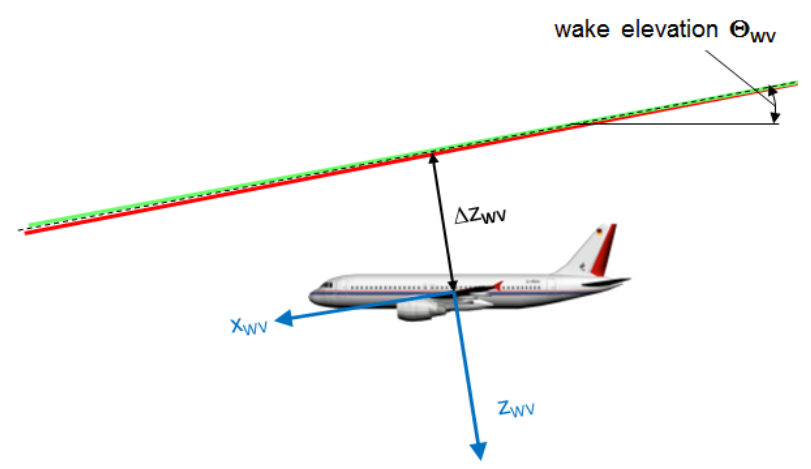

Figure 3. Geometry of LiDAR output parameters (side view). wake vortex encounter, however, the relative angle between the wake vortex axis and the flight path of the aircraft is rather small. The main components of the wake vortex velocities consequently occur perpendicular to the measurement direction and are not detected by a pure line of sight measurement (i.e. along the laser beam). In order to use the LiDAR measurement for the wake impact alleviation a wake identification algorithm is included in the concept as shown in Fig. 1. The algorithm identifies the parameters of a wake vortex model that matches the measured LoS wind components best. Details about the wake identification algorithm will be explained in Section A and can be found in Ref. 9 and Ref. 10. The wake vortex model is an analytical Burnham-Hallock model ${ }^{11}$ and is defined by the characteristic wake vortex parameters vortex circulation $\Gamma$, lateral separation of the vortex cores b', wake elevation $\Psi_{\mathrm{wv}}$ and azimuth $\Theta_{\mathrm{wv}}$ and the lateral and vertical distance of the wake vortex from the vortex centerline to the center of gravity of the aircraft $\Delta \mathrm{y}_{\mathrm{wv}}$ and $\Delta \mathrm{z}_{\mathrm{wv}}$ as illustrated in Fig. 2 and 3.

Once the wake identification algorithm has converged to a valid solution, which is verified by a plausibility check of the identified parameters, the parameters are passed to the wake impact alleviation controller. The identified position parameters are stored in a north-east-down (NED) coordinate system whose origin is located at the center of gravity of the aircraft. The benefit of this aircraft-carried coordinate system is that the influence of stationary wind which

only shifts the aircraft and the wake vortex parallelly, but does not cause any change in the location of the wake vortex relative to the aircraft is avoided. As the position of the wake vortex is defined relative to the aircraft position, it needs to be updated when the aircraft moves relative to the surrounding air. This is realized by shifting the wake vortex position by the change of the aircraft position relative to the surrounding air. The latter can be derived by transforming the body-fixed components of the true airspeed into the NED coordinate system and integrating these components. The integration of airspeed components in this process is approximated by a multiplication with the simulation sample time:

with $\Delta t_{s}=$ simulation sample time [sec]

$$
\left[\begin{array}{l}
x \\
y \\
z
\end{array}\right]_{N E D, \text { new }}=\left[\begin{array}{l}
x \\
y \\
z
\end{array}\right]_{N E D, \text { old }}-\left[\begin{array}{c}
\mathrm{u}_{\mathrm{TAS}} \\
\mathrm{v}_{\mathrm{TAS}} \\
\mathrm{w}_{\mathrm{TAS}}
\end{array}\right]_{N E D} \cdot \Delta t_{S}
$$




$$
\begin{gathered}
{\left[\begin{array}{c}
\mathrm{u}_{\mathrm{TAS}} \\
\mathrm{v}_{\mathrm{TAS}} \\
\mathrm{w}_{\mathrm{TAS}}
\end{array}\right]_{N E D}=\text { components of true airspeed in NED coordinates }} \\
\qquad{\underline{M_{N E D, b}}} \cdot V_{T A S}\left[\begin{array}{c}
\cos \alpha_{A} \cdot \cos \beta_{A} \\
\sin \beta_{A} \\
\sin \alpha_{A} \cdot \cos \beta_{A}
\end{array}\right]
\end{gathered}
$$

whereupon

$$
\underline{M}_{N E D, b}=\left[\begin{array}{ccc}
\cos \Psi \cos \Theta & \cos \Psi \sin \Theta \sin \Phi-\sin \Psi \cos \Theta & \cos \Psi \sin \Theta \cos \Phi+\sin \Psi \sin \Theta \\
\sin \Psi \cos \Theta & \sin \Psi \sin \Theta \sin \Phi+\cos \Psi \cos \Phi & \sin \Psi \sin \Theta \cos \Phi-\cos \Psi \sin \Phi \\
-\sin \Theta & \cos \Theta \cos \Phi & \cos \Theta \cos \Phi
\end{array}\right] .
$$

Concerning the aerodynamic angle of attack $\alpha_{A}$ and sideslip $\beta_{A}$ and the true airspeed $V_{\text {TAS }}$ it is assumed that these parameters are filtered in that way that they represent values relative to the stationary surrounding air and do not contain unsteady wind components of the wake vortex. The stored wake vortex parameters represent the basis for the determination of the disturbance wind velocities at the strips of the aerodynamic interaction model (AIM). In order to account for the time delay due to processing time and actuator delays, the position at which the wind velocity is calculated, is not the current position, but the estimated position of each strip after this known time delay. The prediction of the strip positions is realized as a propagation of the current position on the basis of the current translational and rotational velocity. These are approximated as:

$$
\begin{aligned}
& {\left[\begin{array}{c}
\Phi \\
\Theta \\
\Psi
\end{array}\right]_{\text {pred }}=\left[\begin{array}{c}
\Phi \\
\Theta \\
\Psi
\end{array}\right]+\left[\begin{array}{c}
\dot{\Phi} \\
\dot{\Theta} \\
\dot{\Psi}
\end{array}\right] \cdot \Delta t_{\text {delay }},} \\
& {\left[\begin{array}{c}
\Delta x \\
\Delta y \\
\Delta z
\end{array}\right]_{N E D}=\left[\begin{array}{c}
\mathrm{u}_{\mathrm{TAS}} \\
\mathrm{v}_{\mathrm{TAS}} \\
\mathrm{w}_{\mathrm{TAS}}
\end{array}\right]_{N E D} \cdot \Delta t_{\text {delay }}}
\end{aligned}
$$

with $\Delta t_{\text {delay }}=$ time delay between control deflection command generation and command realization.

Once the predicted position of each AIM strip is determined for the moment when the command control surface deflection will be achieved, the velocity at each strip is calculated by applying a Burnham-Hallock wake vortex model using the identified characteristic wake vortex parameters. Given the velocities at the strips the AIM can be used to derive the wake-induced disturbances moments acting on the aircraft.

The AIM is a way to model the interaction of a wake vortex with an aircraft by means of delta wake-induced aerodynamic forces and moments, based on lifting line theory. The different components of the aircraft are divided into strips. For each strip the additional angles of attack (wing, horizontal tail) and angles of sideslip (vertical tail, fuselage) and the resulting additionally induced forces and moments are computed due to the local wind of the wake vortex. The overall wake-induced forces and moments are derived by summing up the incremental forces and moments of each strip. Further details about the AIM model and its validation can be found in Ref. 12.

When the wake-induced forces and moments are known, the idea is to command the corresponding control surface deflections that generate exactly the negative of the wake-induced forces and moments such that no wakeinduced aircraft response occurs. Unfortunately for this approach, common aircraft configurations only exhibit control surfaces in three degrees of freedom (roll, pitch, and yaw) whereas the wake disturbance generally acts in all six degrees of freedom. It is thus not possible to compensate for all wake-induced forces and moments with the ailerons, elevator and rudder at each point in time. Therefore, the approach chosen here is to compensate for the three wake-induced moments. The required control surface deflections to counteract these moments are determined by inverting the aerodynamic model of the aircraft. This leads to the following control surface deflections for the aileron and rudder:

$$
\left[\begin{array}{c}
\Delta \delta_{\text {ail }} \\
\Delta \delta_{\text {rud }}
\end{array}\right]=-\frac{s}{\bar{q} \cdot S} \cdot\left[\begin{array}{ll}
\left(C_{l, \delta_{\text {ail }}}(\vec{y})+\sum_{i=2}^{5} K_{\delta_{\text {ail }} \delta_{S P} .} C_{l, \delta_{S P, i}}(\vec{y})\right) & C_{l, \delta_{\text {rud }}}(\vec{y}) \\
\left(C_{n, \delta_{\text {ail }}}(\vec{y})+\sum_{i=2}^{5} K_{\delta_{\text {ail }} \delta_{S P}} \cdot C_{n, \delta_{S P, i}}(\vec{y})\right) & C_{n, \delta_{\text {rud }}}(\vec{y})
\end{array}\right]^{-1}\left[\begin{array}{c}
L_{W V} \\
N_{W V}
\end{array}\right] .
$$

It is assumed here that the control allocation equations are linear in the control surface deflections. Generally the control surface efficiencies, such as $C_{l, \delta_{a i l}}$ and $C_{l, \delta_{r u d}}$, are nonlinear functions of the model output vector $\vec{y}$. 
The aircraft used is equipped with 5 pairs of spoilers whereupon spoilers 2 to 5 are used as roll spoilers. They are connected to the aileron command via the fixed factor $K_{\boldsymbol{\delta}_{\boldsymbol{a i l}} \boldsymbol{\delta}_{\boldsymbol{S P}}}$ which defines the ratio of the spoiler deflection difference of each spoiler pair (left-right) to the aileron deflection. It is thereby assumed that the same differential command is generated for each spoiler pair.

If an aileron deflection $\Delta \delta_{\text {ail, } R}$ has been determined for the wake vortex disturbance rejection the following additional spoiler deflections will be commanded by the wake impact alleviation function:

$$
\left[\begin{array}{c}
\Delta \delta_{S P, 2, L-R} \\
\cdots \\
\Delta \delta_{S P, 5, L-R}
\end{array}\right]=\left[\begin{array}{c}
K_{\delta_{\text {ail }} \delta_{S P}} \\
\cdots \\
K_{\delta_{\text {ail }} \delta_{S P}}
\end{array}\right] \Delta \delta_{\text {ail }, R} .
$$

As the contribution of the spoiler deflection on the pitching moment cannot be neglected, this additional pitching moment has to be considered in the solution of the required elevator deflection. Therefore, the elevator has to compensate the wake-vortex-induced pitching moment as well as the pitching moment generated by the spoiler deflection.

$$
\Delta \delta_{\text {elev }}=-\frac{l_{\mu}}{\bar{q} \cdot S \cdot C_{m, \delta_{\text {elev }}}(\vec{y})} \cdot\left(M_{W V}+M_{S P}\right)
$$

Considering the pitching moment equation, the assumption that the control allocation equations are linear in the control surface deflection leads to a negligence of the pitching moment due to the induced drag contribution of the elevator deflection. This is acceptable, because the contribution of the elevator deflection to the induced drag and the contribution of the induced drag to the overall pitching moment are negligible.

The additional control deflection of the aileron, roll spoilers, rudder and elevator are added to the current control surface deflections commanded by the pilot or the regular flight control system. This way, the wake-induced aircraft response shall be alleviated by eliminating the rotational disturbance reaction due to the wake vortex.

The simulation model used for the analyses in this paper include a representative Fly-by-Wire augmented manual control system, whose control commands are added to the WIAC commands. Basically, the control system uses a load factor command system in the pitch axis, a roll rate command / bank angle hold system in the roll axis and a sideslip angle command in the yaw axis. The sideslip angle is estimated using lateral acceleration measurements. The lateral control system, consisting of the roll and yaw control loops, is designed to decouple the roll and yaw motion in presence of external disturbances, as described in Ref. 13. For the analysis presented it is assumed that the pilot does not generate any stick or pedal inputs. The basic control system thus attempts to compensate for any deviation of the vertical load factor and to keep the roll rate and sideslip angle equal to zero. Section III and IV show the success of this approach under ideal and realistic conditions, respectively.

\section{A. Description of Wake Identification Algorithm}

As explained before most of the information on the wake vortex is lost if a line-of-sight LiDAR measurement is used. A processing of the data is thus essential in order to use the LiDAR measurement for a wake impact alleviation control system. The method used for this processing is the "Online Wake Identification (OWI)", shown in Fig. 4, where the parameters of a wake model are approximated before the aircraft encounters the wake.,

A key feature of the OWI is the model-based reconstruction of the LIDAR LoS-velocities, which are compared and adapted ("Comparator", Fig. 4) to the measured ones applying the maximum likelihood cost function. In addition to the LiDAR line-of-sight measurements, further OWI inputs include measurements of the aircraft position and the aircraft Euler angles. As a result the OWI outputs the seven model parameters which describe the applied wake model. With this model the wake flow field is approximated in the geodetic coordinate system. In order to find the best (i.e. most likely) wake model parameter values, the product of the error variances of the LoS-velocities is minimized using a Gauss-Newton algorithm. Defining the cost function as the product of the error variances is an approximation of the covariance matrix determinant for a diagonally dominant matrix. Details on the maximum likelihood estimation used in this paper can be found in Ref. 14 (Chapter 4 "Output Error Method", section V.B "Unknown Measurement Noise Covariance Matrix"). In this process, not only is the single-time line-of-sight measurement snapshot interpreted but the time histories of the measurements. The time window used for evaluation is of finite length (e.g. $10 \mathrm{~s}$ ) and is permanently updated if new measurement information is available. The OWI algorithm works iteratively, so an initial guess for the model parameters is necessary. Five of the parameters are initialized autonomously OWI/aircraft. The two parameters concerning the wake orientation are initialized using information from a potential generator aircraft flying in the surrounding area via the standard ADS-B. 


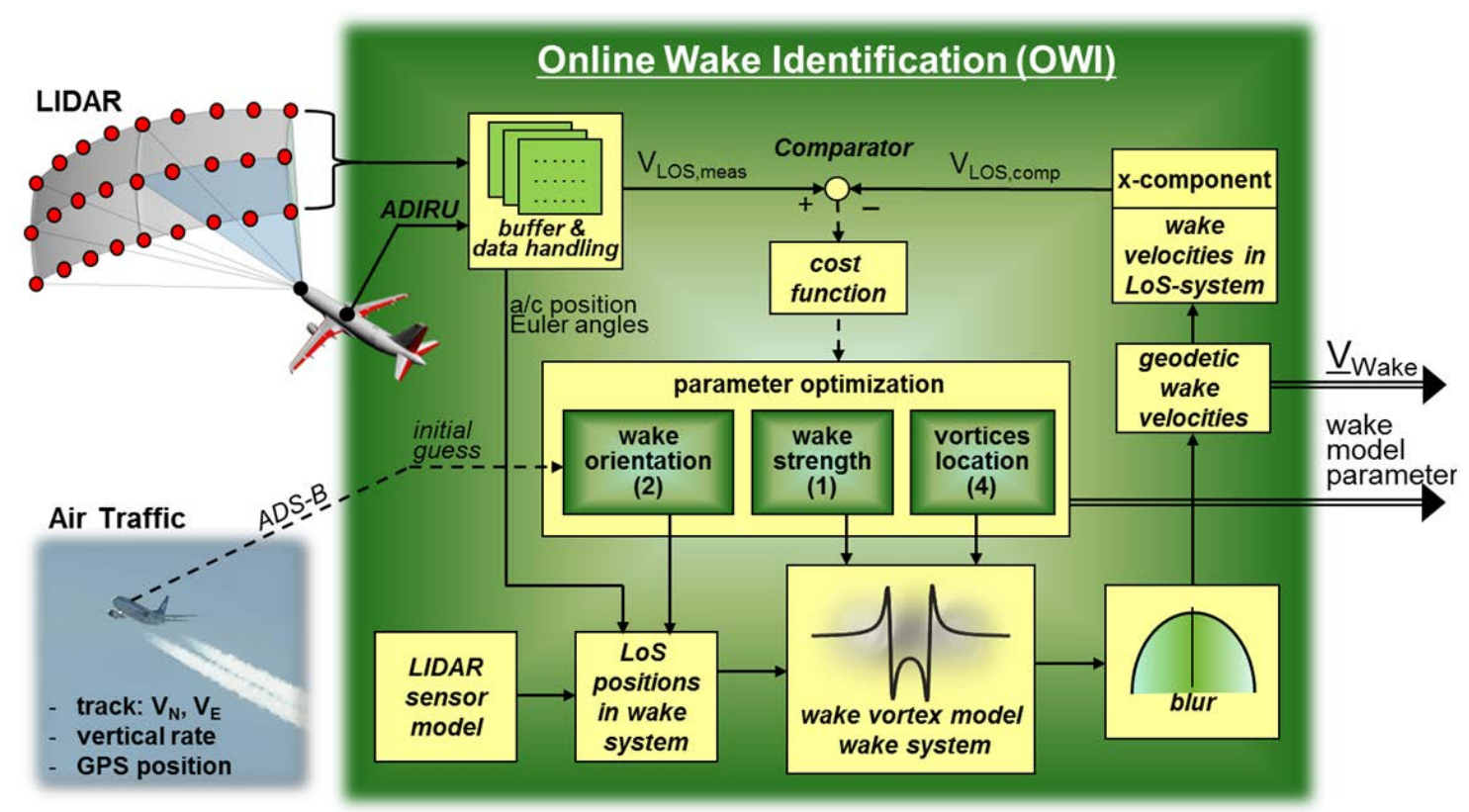

Figure 4. Principle of the Online Wake Identification (OWI).

To apply the OWI method successfully, an appropriate LoS array, laser power, and update rate are essential. Ideally, high measurement quality is desired with a low laser power, a low LoS update rate, and a low number of LoS-directions. A suitable LoS array includes high cross-eyed LoS angles which provides measurements at positions clearly beyond the aircraft dimensions. This is important for gathering maximum measurement information of a wake before entry. Furthermore, high cross-eyed angles also measure considerable components of the wake velocities for small (and dangerous) encounter angles. A suitable LiDAR arrangement should also provide sufficiently small angles between two nearby LoS-directions. This is to get sufficient measurement information within the dimension of a wake. So, an appropriate LiDAR arrangement depends besides the LiDAR capability on the aircraft geometry, measurement range ahead, airspeed, and the typical dimension of HEAVY (and upper MEDIUM) types of wake generators. An example
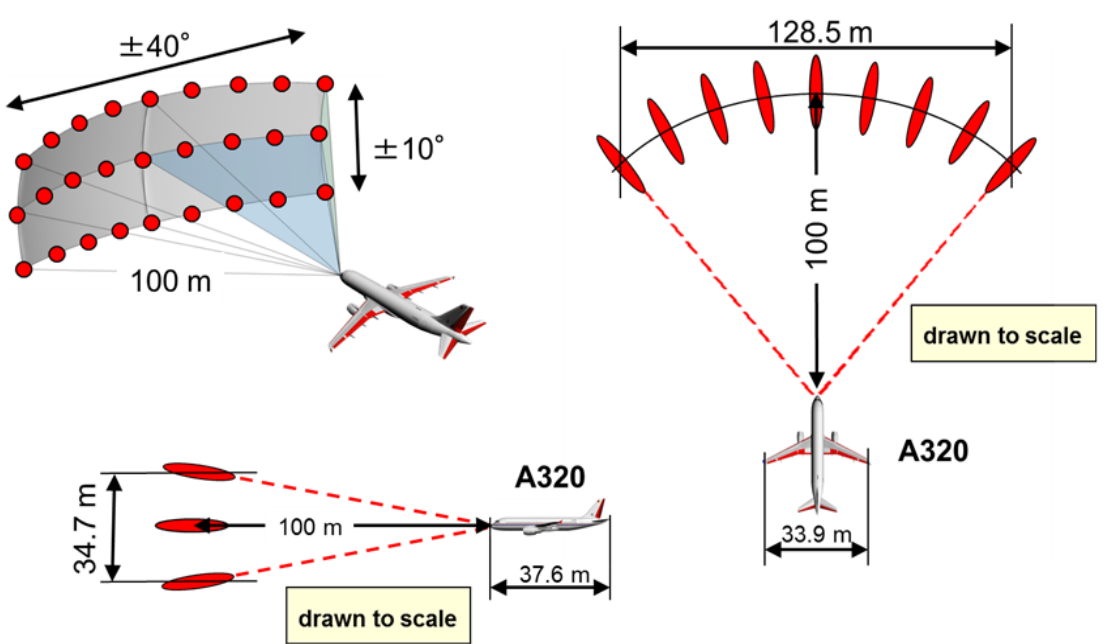

Figure 5. Drawn to scale sketch of a 3x9 LIDAR configuration on an A320 with $100 \mathrm{~m}$ nominal measurement range.

for a suitable LiDAR array for an A320 aircraft is a 3x9 configuration, as shown in Fig. 5, with a measurement range of nominal $100 \mathrm{~m}$ in front of the aircraft nose. ${ }^{9} 27 \mathrm{LoS}$ directions are arranged in a symmetric, equidistant scan pattern: $\pm 40^{\circ}$ in lateral direction, $\pm 10^{\circ}$ in vertical direction, which provide measurements between $65 \mathrm{~m}$ left and right of the aircraft nose, (about factor 4 of the A320's half wing span). This enables measurement information also for quasi-parallel and dangerous encounters (limit scenario). The vertical measurement range is about $35 \mathrm{~m}$ for a 3x9 configuration. Other scan patterns within these ranges are applicable as well for the OWI algorithm. 


\section{Success of WIAC in Case of Ideally Known Wake Vortex Parameters}

In this section the potential of the wake impact alleviation approach is analyzed for the idealized case that the wake vortex parameters, on the basis of which the wake disturbance is derived, are ideally known. This corresponds to the case when the wake identification delivers a perfect match of the wake vortex model with the actual disturbance. In reality this ideal match is not achievable, because, even if the decay process of a wake vortex has not advanced very much a realistic wake vortex never looks exactly like an analytical Burnham-Hallock wake vortex. Nevertheless, the assumption of perfectly identified wake vortex parameters allows an analysis of the maximum achievable success of the wake impact alleviation control system. Figure 6 depicts the comparison of the aircraft responses with and without the wake impact alleviation control system for a lateral wake vortex encounter with $15^{\circ}$ lateral and $0^{\circ}$ vertical encounter angle whereupon the wake vortex is located $2 \mathrm{~m}$ above the aircraft. The wake vortex disturbance acting on the aircraft here is modeled with the same Burnham-Hallock model which is used inside the wake impact alleviation function. The encounter scenario corresponds to a A320 like aircraft encountering the wake vortex of a A340 size aircraft during approach. The interaction of the wake vortex with the aircraft is modeled by an Aerodynamic Interaction Model (AIM) as described in Section II and in Ref. 12. The wake-induced drag is not considered in this model. However, analyses in Ref. 12 showed that the applied AIM appropriately models the interaction of the wake vortex with the aircraft. In the present case, there are no pilot inputs or autopilot commands applied during the encounter. Only the basic augmented manual control system, and when indicated, the wake impact alleviation system is active. The red and the blue line show the same encounter whereupon the red line corresponds to the case without the application of the wake impact alleviation controller and the blue dashed line depicts the aircraft response when the wake impact alleviation control system is active. The figure shows that the wake-induced attitude change can be significantly reduced by means of the wake impact alleviation controller. The maximum bank angle during the encounter can be reduced from $8.9^{\circ}$ to $2.7^{\circ}$ and the pitch angle and heading deviation are also remarkably decreased.
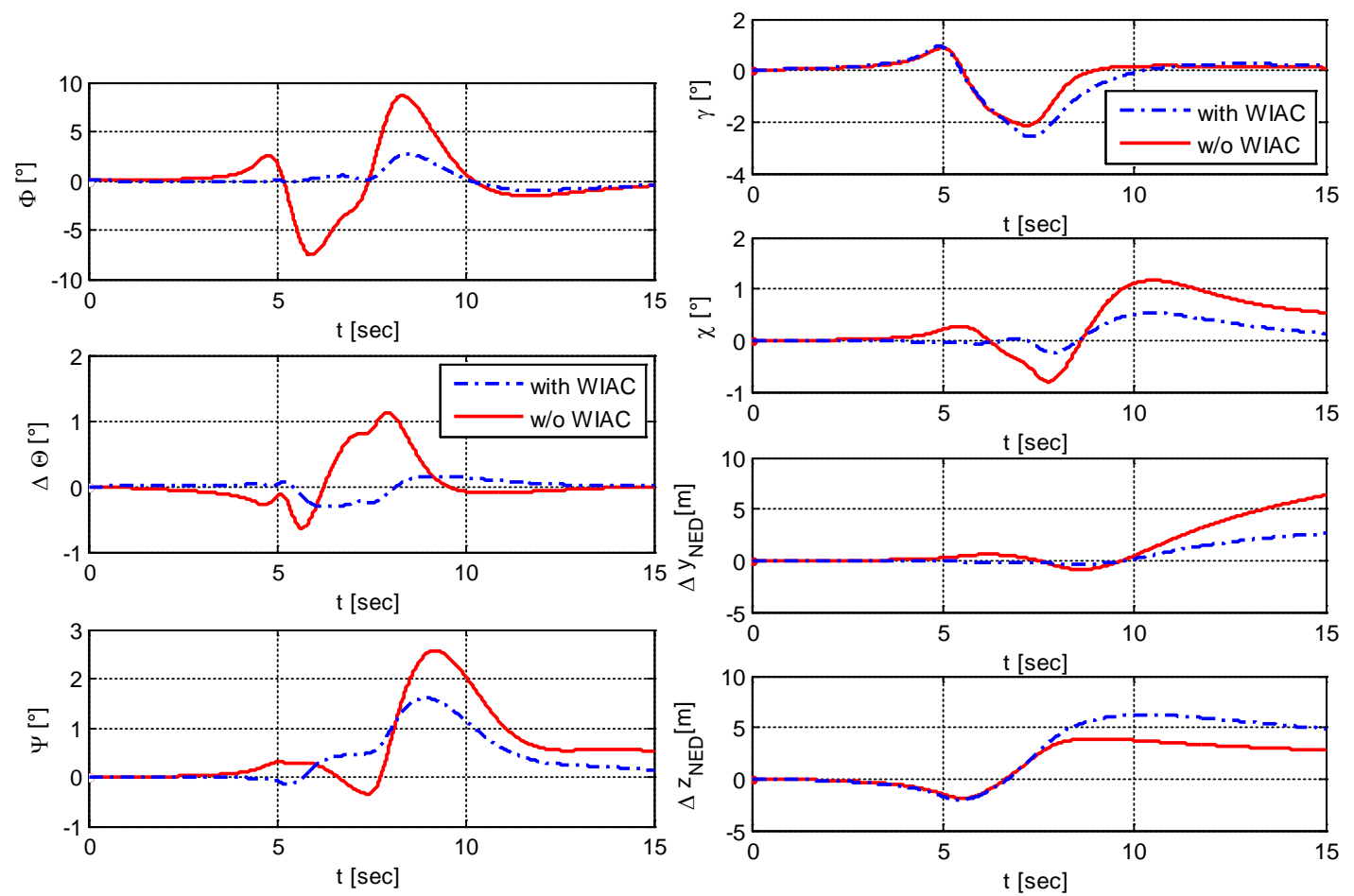

Figure 6. Achievable wake impact alleviation in case of ideally known wake vortex parameters.

At first sight the observation that there are still small attitude changes even though the disturbance is ideally known seems surprising. It might be expected that the approach of commanding the control surface deflections corresponding to the negative of the wake-induced moments should completely eliminate all attitude changes. However, it has to be kept in mind that the basic control system of the aircraft is still active and adds control surface commands to the WIAC outputs. Furthermore, the approach taken here only considers the input matrix for the determination of the control commands and does not take into account changes of aircraft states. If there are strong changes in some aircraft states, which can occur because the available control surfaces can only influence three 
degrees of freedom (roll, pitch, and yaw) whereas the wake disturbance generally acts in all six degrees of freedom, this has an influence on the computation of the required control surface deflection. It is assumed here that the state changes are small. The rotational rates should be approximately constant because the disturbance moments are compensated for by the wake impact alleviation control commands. An important parameter that is not influenced by the WIAC is the angle of attack (or the vertical velocity w respectively). If there are for instance strong upwinds or downdrafts during the wake encounter this can lead to angle of attack changes that cannot be neglected for the computation of the required control surface deflections. In the case of the considered aircraft, however, the basic control system for the longitudinal motion is design as an $\mathrm{n}_{\mathrm{z}}$-control law which attempts to keep the vertical load factor constant. It has been found that thanks to this reaction of the basic control system the changes in the angle of attack do not exhibit large variations during the wake encounter, which leads to still acceptable commands of the WIAC. The approach taken here to compute the control surface deflection by inverting the input matrix only without considering angle of attack changes is thus still feasible. It is, however, one of the influences that explain the fact that the attitude change is not completely zero when the wake impact alleviation control system is applied on the basis of ideally known wake vortex parameters.

The comparison of the lateral flight path deviation $\Delta \mathrm{y}_{\mathrm{NED}}$ and track excursion $\chi$ on the right-hand side of Fig. 6 shows that the wake impact alleviation control system leads to significantly reduced deviations of the lateral flight path. For the vertical flight path, however, larger deviations can be observed when the wake impact alleviation is active than in the case without the application of this system. This effect occurs because the WIAC only compensates for the rotational disturbance without considering the vertical wake-induced disturbance. In the case of the presented encounter, the wake vortex induces a pitch-up and a vertical downwards motion at the same time. A pitch-up rotation alone would lead to a negative deviation from the target z-position in NED coordinates (i.e. above the target flight path) and thus counteracts the downdraft of the wake vortex. If the wake impact alleviation controller prevents the wake-induced pitching moment the vertical flight path deviation is increased compared to the case without the application of the WIAC. As the increased flight path deviation is in a downward direction this effect might be critical if the encounter occurs close to the ground. This effect indicates that the current purpose of the wake impact alleviation system to only compensate for rotational disturbances might have to be revised. It is imaginable that an alleviation of a combination of the pitch-up motion and the vertical flight path deviation would be preferable in this encounter situation. Research is still necessary with respect to the exact goal of the wake impact alleviation, i.e. the deviation of which parameters or parameter combinations should ideally be minimized.

\section{Success of WIAC in Combination with Realistic OWI Results}

After the potential of the wake impact alleviation concept has been assessed for the case of a perfect identification of the wake vortex disturbance in section III, the success of the wake impact alleviation controller shall be analyzed for the application in combination with the online wake identification algorithm. For this purpose the same encounter scenario as in Section III is studied for the case that the vortex parameters of the BurnhamHallock model within the WIAC algorithm originate from the wake identification.

As the LiDAR sensor has a limited measurement range, the OWI only converges to a valid result when the aircraft is close enough to the wake vortex and the LiDAR sensor provides appropriate measurements. Once the wake identification has converged to a valid result, the identified parameters are passed to the alleviation control system and the latter starts to counteract the wake vortex disturbance. The presented simulations of wake vortex encounters start at the moment when the identified wake alleviation function is activated. The aircraft is trimmed out at the beginning of the simulation which is a simplification as the wake vortex could theoretically already lead to small deviations from the trimmed flight condition at this time. However, as a good result of the wake identification is already available at a considerable distance before the wake vortex, the deviation from the trimmed flight condition can be assumed to be small at this point.

Table 1. Error of identified parameters for $15^{\circ}$ lateral encounter.

\begin{tabular}{|l|c|}
\hline Parameter & Error \\
\hline Circulation $\Gamma\left[\mathrm{m}^{2} / \mathrm{s}\right]$ & -15.62 \\
\hline Lateral vortex separation $\mathrm{b}^{\mathrm{c}}[\mathrm{m}]$ & 0.54 \\
\hline Wake azimuth $\left.\Psi_{\mathrm{wv}}{ }^{\circ}\right]$ & 0.01 \\
\hline Wake elevation $\Theta_{\mathrm{wv}}{ }^{\circ}{ }^{\circ}$ & 0.17 \\
\hline $\begin{array}{l}\text { y-distance from CG of aircraft } \Delta \mathrm{y}_{\mathrm{wV}}[\mathrm{m}] \\
\text { (cf. Fig. 2) }\end{array}$ & -2.95 \\
\hline $\begin{array}{l}\text { z-distance from CG of aircraft } \Delta \mathrm{z}_{\mathrm{WV}}[\mathrm{m}] \\
\text { (cf. Fig. 3) }\end{array}$ & 0.97 \\
\hline
\end{tabular}




\section{A. Attainable Alleviation for $15^{\circ}$ Lateral Encounter}

The application of the wake impact control system in combination with the wake identification is assessed for the encounter scenario of Section III with a vertical offset between the wake vortex and the aircraft. This is a $15^{\circ}$ lateral encounter of a A320 size aircraft behind a A340 size aircraft in approach whereupon the wake vortex is located $2 \mathrm{~m}$ above the center of gravity of the aircraft. The wake vortex is modeled as an analytical Burnham-Hallock wake vortex. The LiDAR sensor, which is used for the wind velocity measurement in this case, has an average laser power of $25 \mathrm{~W}$ and 27 measurement directions with a point to point update rate of $270 \mathrm{~Hz}$. Each measurement direction has one measurement point at a fixed range of $100 \mathrm{~m}$. The measurement points are arranged in a rectangular pattern with 9 horizontal and 3 vertical points and a horizontal scan angle of
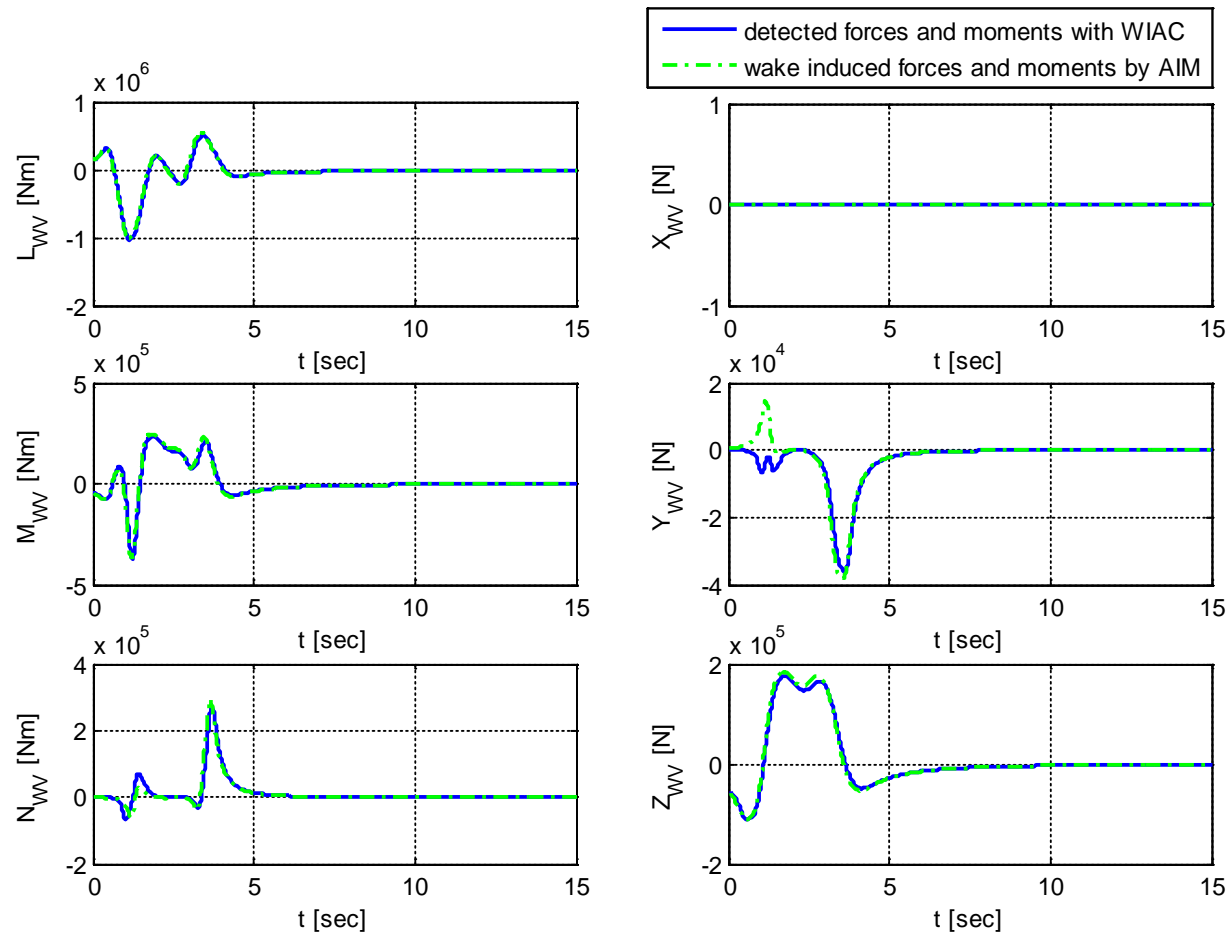

Figure 7. Actual wake-induced and detected forces and moments for $15^{\circ}$ lateral encounter.

$+/-40^{\circ}$ and a vertical scan angle of $+/-10^{\circ}$. These LiDAR parameters are relatively optimistic compared to current LiDAR technology standards. Nevertheless, they are chosen in order to assess the general feasibility of the combined application of the OWI and wake impact alleviation control system.

Table 1 shows the precision of the wake vortex parameters that can be achieved with the wake identification for this LiDAR characteristics, whereupon a positive error indicates that the identified parameter is larger than the true value. The occurring deviation from the true parameters of the applied wake vortex model is very small. In particular the orientation of the wake vortex is matched very well. Only the circulation exhibits a noticeable error of $-15.62 \mathrm{~m}^{2} / \mathrm{s}$ from the actual vortex strength of $340.66 \mathrm{~m}^{2} / \mathrm{s}$. However, as the vortex circulation only influences the magnitude of the disturbance velocities but does not affect the location or direction of the wind velocities the consequence of an error in this parameter is comparably low.

Figure 7 illustrates the comparison of the actual wakeinduced forces and moments of the wake vortex model acting on the aircraft (green dashed line) and the disturbance forces and moments detected by the WIAC on the basis of the identified wake vortex parameters (blue line). Despite the small errors of
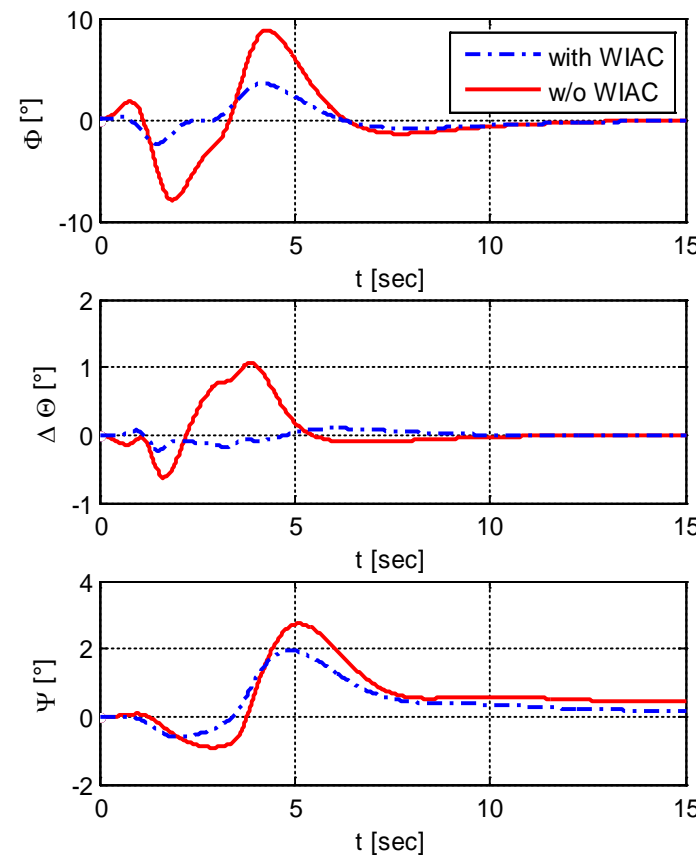

Figure 8. Attitude change alleviation for $15^{\circ}$ lateral encounter based on actual wake identification result. 
the identified parameters shown in Table 1, the disturbance forces and moments resulting from the detected wake vortex almost perfectly match the actual wake-induced forces and moments. Only the side force and the yaw moment show small discrepancies between the actual and the detected disturbance at the beginning of the encounter. But the error is still comparably small and only occurs for a short time during the encounter. The wake-induced xforce is zero because the AIM does not model wake-induced drag.

In Fig. 8 the achieved alleviation of the aircraft attitude change resulting from the detected moments of Fig. 7 are presented. It can be seen that the wake impact alleviation works very well and significantly reduces the wakeinduced changes of the Euler angles, if it is applied in combination with the wake identification algorithm. Even though the identified wake vortex parameters and the resulting detected wake-induced moments exhibit small errors, the success of the reduction of the attitude changes is barely impaired compared to the case when the wake parameters are perfectly known. For the $15^{\circ}$ lateral encounter with a $2 \mathrm{~m}$ vertical offset between the wake vortex and the aircraft, the maximum bank angle can be reduced from $8.8^{\circ}$ to $3.6^{\circ}$ and the maximum yaw angle is reduced from $2.7^{\circ}$ to $2.0^{\circ}$. The pitch angle change is almost completely eliminated when the wake impact alleviation control system is active.

The observation that the Euler angles without WIAC in Fig. 8 are not identical to the Euler angles without WIAC in Fig. 6 even though both cases have the same encounter angles results from the fact that the starting point of the two encounters differs. In Fig. 6 the wake vortex is located $500 \mathrm{~m}$ ahead of the aircraft when the simulation is started. The simulation presented in Fig. 8 starts when the OWI provides a valid result. The aircraft thus starts the encounter in a trimmed flight condition approximately $180 \mathrm{~m}$ away from the wake vortex. In the encounter scenario of Fig. 6 the wake vortex has already influenced the flight path and attitude of the aircraft at the time the wake vortex in only $180 \mathrm{~m}$ ahead.

\section{B. Influence of Encounter Angle}

Once the wake identification based wake impact alleviation has demonstrated satisfactory results for the reference scenario with a $15^{\circ}$ lateral encounter, the influence of the encounter angle shall be assessed.

Figure 9 presents the achieved alleviation of the Euler angles for the same encounter scenario but with modified lateral encounter angles of $5^{\circ}$ and $35^{\circ}$. In the case of the very shallow encounter angle of $5^{\circ}$ the alleviation of the
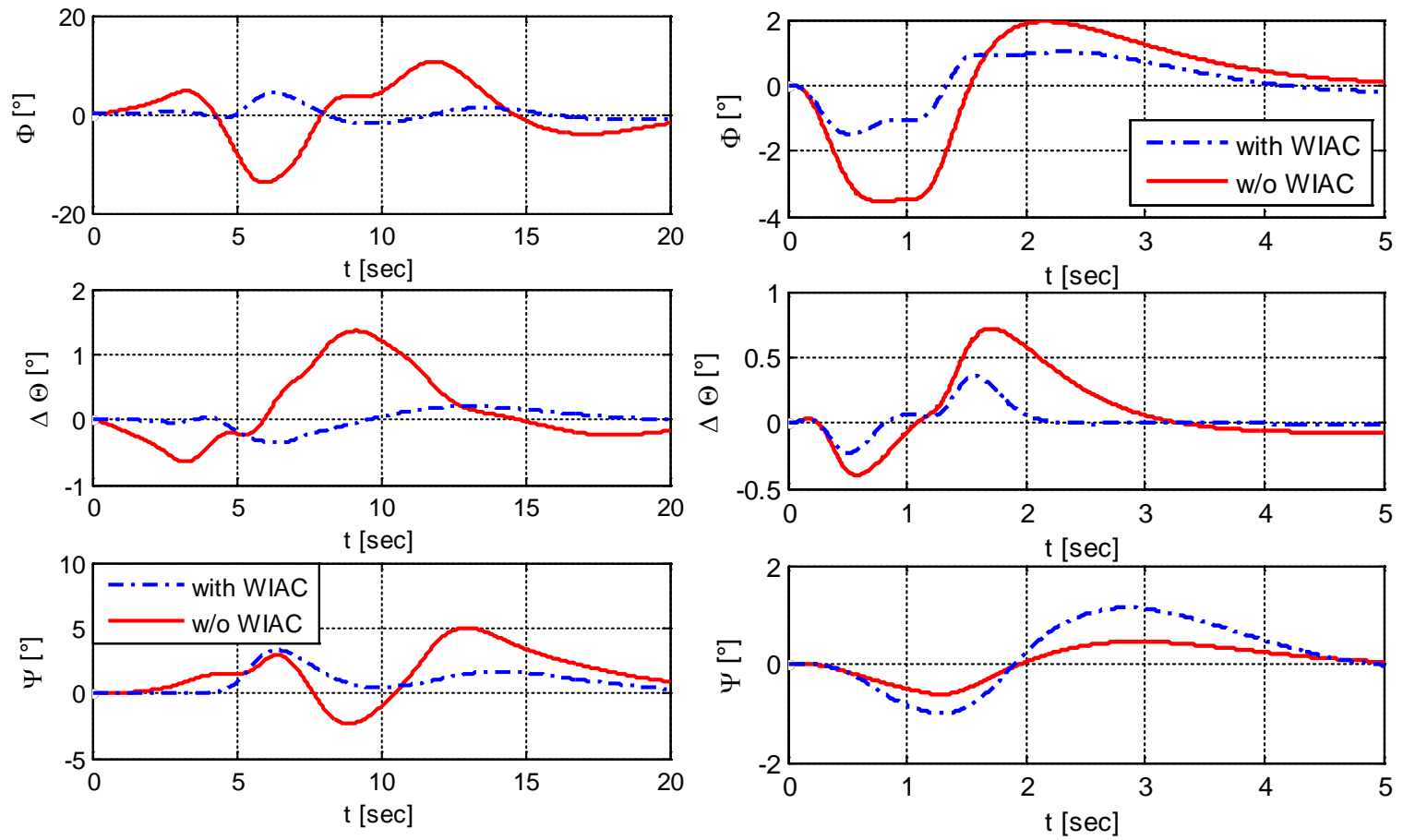

Figure 9. Attitude change alleviation for $5^{\circ}$ (left) and $35^{\circ}$ (right) lateral encounter based on actual wake identification result. 
attitude change works well. The maximum bank angle can be reduced from $13.8^{\circ}$ to $4.3^{\circ}$ and the maximum heading change decreases from $5.0^{\circ}$ to $3.3^{\circ}$. The pitch angle change is again almost completely prevented.

In contrast for the case of the comparably steep encounter with a lateral encounter angle of $35^{\circ}$, the wake impact alleviation does not work as well. For this encounter geometry the results of the wake identification are less exact. For the $y$ and $\mathrm{z}$ - position of the wake vortex significant deviations of more than $6 \mathrm{~m}$ were exhibited. Consequently, the match of the detected wake-induced moments which are used to derive the required control surface deflections is less exact as is shown in Fig. 10. As a consequence the bank and pitch angle are only slightly improved by the wake impact alleviation control system and the yaw angle is even slightly increased. The alleviation is thus not successful for this relatively steep lateral encounter scenario. It has to be noticed, though, that the overall wake-induced attitude changes are in general very small here and stay below $2^{\circ}$ for all Euler angles when the WIAC is active. The encounter can thus be classified as uncritical.
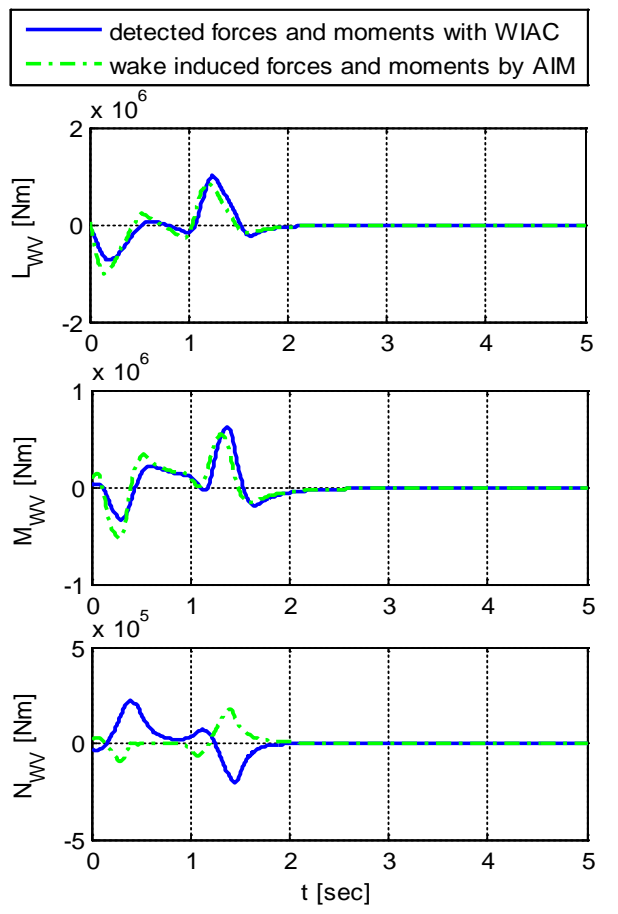

Figure 10. Actual wake-induced and detected forces and moments for $35^{\circ}$ lateral encounter.

\section{Influence of LiDAR Performance}

In addition to the geometry of a wake vortex encounter, another important influence factor for the success of the wake impact alleviation is the performance of the LiDAR sensor. Better sensor characteristics allow an improved measurement of the wind velocities and consequently a better reconstruction of the wake vortex, which shall be compensated for by the wake impact alleviation control system. As the LiDAR sensor characteristics with 25 W, 27 measurement points, and a $270 \mathrm{~Hz}$ update rate are comparably optimistic for the current LiDAR technology, it shall be analyzed how the wake impact alleviation is influenced if the performance of the sensor is reduced. For this purpose the reference encounter scenario of an A320 size aircraft flying with a lateral encounter angle of $15^{\circ}$, no vertical encounter angle, and a vertical offset of $2 \mathrm{~m}$ into the wake vortex of an A340 is assessed for two different characteristics of the LiDAR sensor. In both cases the LiDAR sensor has 9 horizontal and 3 vertical measurement points and again a range of $100 \mathrm{~m}$. The average laser power is reduced from $25 \mathrm{~W}$ to $10 \mathrm{~W}$ and the point to point update rate is reduced from $270 \mathrm{~Hz}$ to $135 \mathrm{~Hz}$. In the first case the scan angles are kept identical to the optimistic LiDAR settings in Section A and B with a horizontal scan angle of $+/-40^{\circ}$ and a vertical scan angle of $+/-10^{\circ}$. For the second LiDAR settings the opening angles of the measurement sphere are reduced to $+/-16^{\circ}$ in the lateral and

Table 2. Error of identified parameters for $15^{\circ}$ lateral encounter with different LiDAR sensors.

\begin{tabular}{|l|c|c|}
\hline \multirow{2}{*}{ Parameter } & \multicolumn{2}{|c|}{ Error } \\
\cline { 2 - 3 } & $\begin{array}{c}\text { reduced LiDAR } \\
\text { performance and } \\
\text { regular scan angles }\end{array}$ & $\begin{array}{c}\text { reduced LiDAR } \\
\text { performance and } \\
\text { small scan angles }\end{array}$ \\
\hline Circulation $\Gamma\left[\mathrm{m}^{2} / \mathrm{s}\right]$ & -29.83 & -1.70 \\
\hline Lateral vortex separation b’ $[\mathrm{m}]$ & -2.53 & 3.12 \\
\hline Wake azimuth $\Psi_{\mathrm{WV}}\left[^{\circ}\right]$ & -0.20 & -0.66 \\
\hline Wake elevation $\Theta_{\mathrm{WV}}\left[^{\circ}\right]$ & 0.34 & 1.42 \\
\hline y-position $\Delta \mathrm{y}_{\mathrm{wV}}\left(\mathrm{cf} . \mathrm{Fig}^{\circ}\right.$ 2) $[\mathrm{m}]$ & -5.41 & -6.44 \\
\hline $\mathrm{z}-$ position $\Delta \mathrm{z}_{\mathrm{WV}}(\mathrm{cf.} \mathrm{Fig.} \mathrm{3)}[\mathrm{m}]$ & 3.61 & 14.86 \\
\hline
\end{tabular}

12 $+/ 8^{\circ}$ in the vertical direction. Table 2 demonstrates the precision of the identified wake vortex parameters for the two LiDAR sensor settings with reduced performance. Compared to the wake identification on the basis of the optimistic LiDAR characteristics in Table 1 , the errors of the wake parameters are increased. The $y$ - and z-position, which have a strong influence on the wake disturbance affecting the aircraft, exhibit larger errors. Due to the reduced laser power the measurement noise increases and the

American Institute of Aeronautics and Astronautics 
measurement of the wind velocities is less exact. As a consequence the wake identification no longer finds the correct wake vortex parameters as precisely. When the scan angles are reduced as well, the error of the identified parameters is further increased. Only the circulation error is smaller than in the case with regular scan angles. The influence of the vortex strength on the wake-induced parameters is, however, comparably low in relation to the influence of the location and orientation of the wake vortex, which are less well identified here.

As a result of the decreased precision of the wake identification, the success of the wake alleviation is also impaired should the LiDAR performance be reduced. Figure 11 illustrates the attainable alleviation of the wake-induced attitude changes for the two LiDAR settings with reduced performance in comparison to the alleviation for the LiDAR sensor with high performance and regular scan angles. While the maximum bank angle is reduced from $8.8^{\circ}$ to $3.6^{\circ}$ in the case of the high performance LiDAR sensor, the magnitude of the bank angle can only be decreased to $4.6^{\circ}$ for the LiDAR sensor with low laser power and regular scan angles; in case of lower laser power and small scan angles to $6.4^{\circ}$. The heading change cannot be reduced when the LiDAR laser power is lower and the pitch angle alleviation is also impaired. The attainable alleviation of the wake-induced attitude change is thus significantly affected if the LiDAR characteristics deteriorate. Nevertheless, the bank and
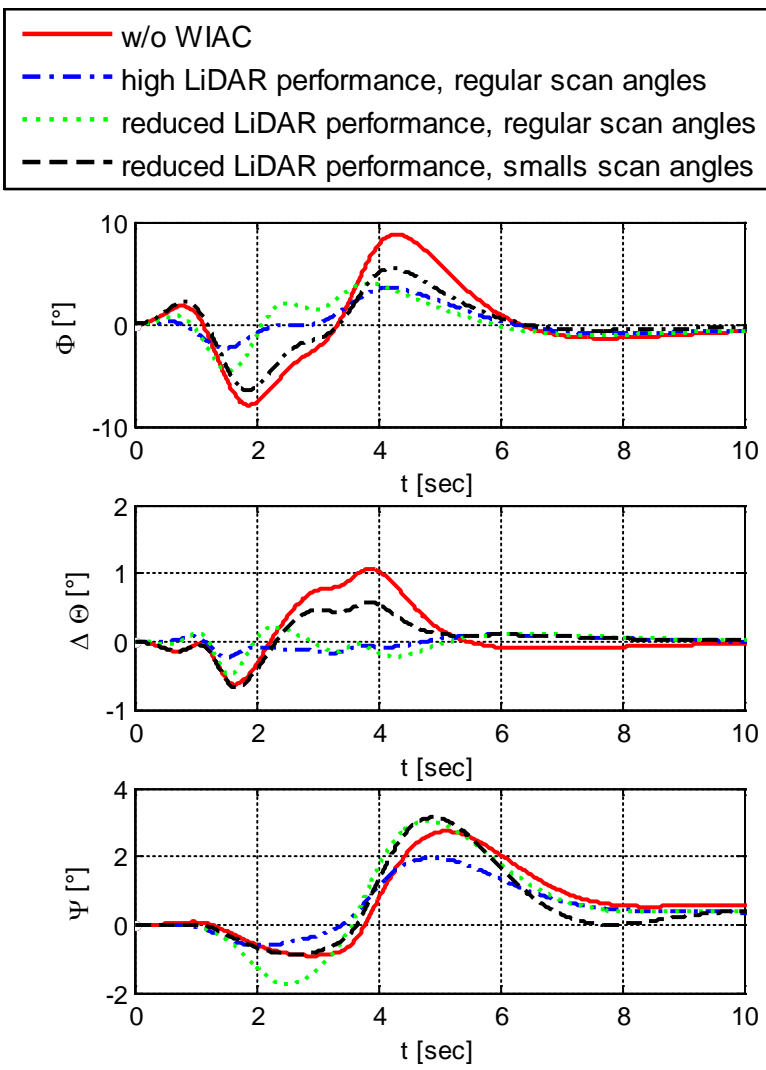

Figure 11. Comparison of attitude change alleviation for different LiDAR characteristics.

pitch angles are still smaller than without the wake impact alleviation and the heading is only slightly increased, but overall small. Therefore, it is still beneficial to apply the wake impact alleviation controller under such conditions.

\section{Sensitivity of Wake Impact Alleviation with Respect to Precision of Identified Wake Vortex Parameters}

Section IV outlined that the quality of the wake identification varies depending on the encounter scenario and the LiDAR characteristics. As the identified wake vortex parameters represent the basis on which the control surface commands to counteract the wake vortex disturbance are derived, the success of the wake impact alleviation is directly influenced by the precision of the identified wake parameters. It is thus important to analyze the sensitivity of the wake impact alleviation with respect to the accuracy of the wake identification. Figure 12 to 15 illustrate the influence of the error of the different wake vortex parameters on the attainable reduction of the maximum bank angle during a wake vortex encounter.

The influence of the error of the identified vortex circulation is relatively low. Even a deviation of $+/-50 \%$ from the true vortex circulation only causes a comparably small impairment of the reduction of the maximum wakeinduced bank angle occurring during the encounter (Fig. 12). As shown in Fig. 13, the benefit of the wake impact alleviation function in the roll axis is already completely eliminated, if an error of -50\% occurs in the identification of the lateral separation of the vortex cores. The vortex circulation only influences the strength of the disturbances velocities but does not change the shape of the disturbance moments. If the vortex circulation is identified too low or too high, the additional control surface deflections commanded by the WIAC will be too small or too large in amplitude but with the correct signs and react at the correct point in time. The lateral separation of the vortex cores, in contrast, also influences the shape of the wake-induced disturbance forces and moments. It is thus plausible that an error in this parameter has a stronger influence on the attainable reduction of the wake-induced bank angle. If the 
additional control surface deflections are derived on the basis of a incorrectly detected time history of the disturbance moments due to faultily identified lateral vortex separation, the deflections might be of incorrect sign, and hence, in the worst case, additionally aggravate the bank angle excursion.

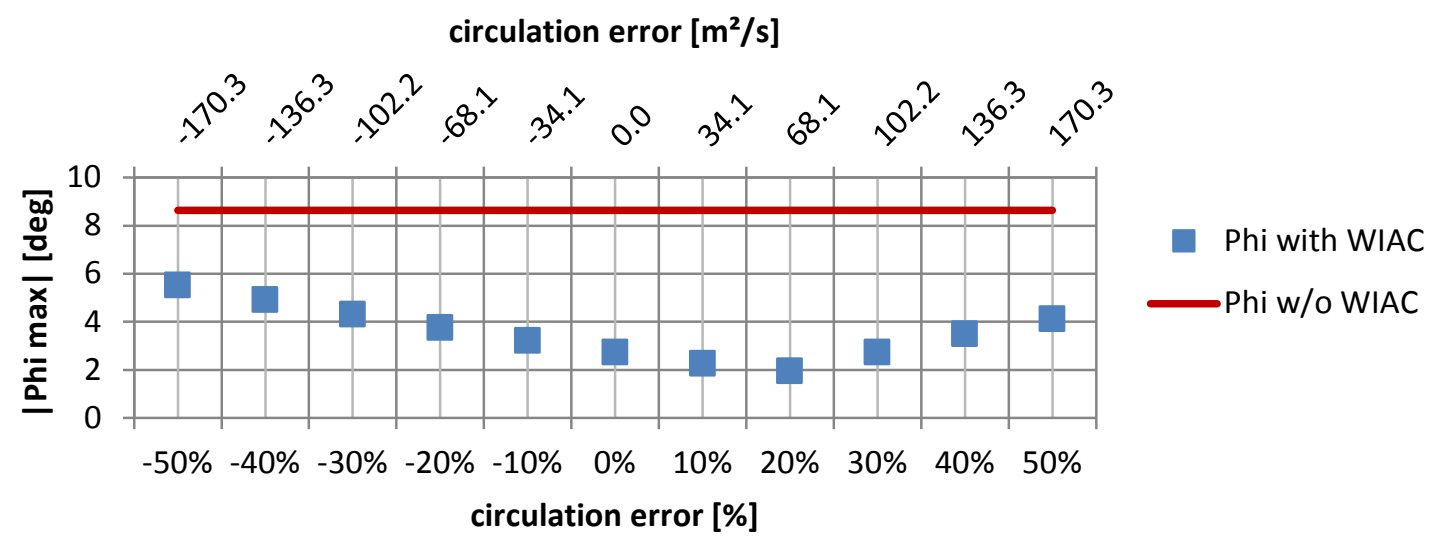

Figure 12. Impairment of reduction of maximum bank angle amplitude by the wake impact alleviation controller due to error of identified vortex circulation.

lateral vortex separation error $[\mathrm{m}]$
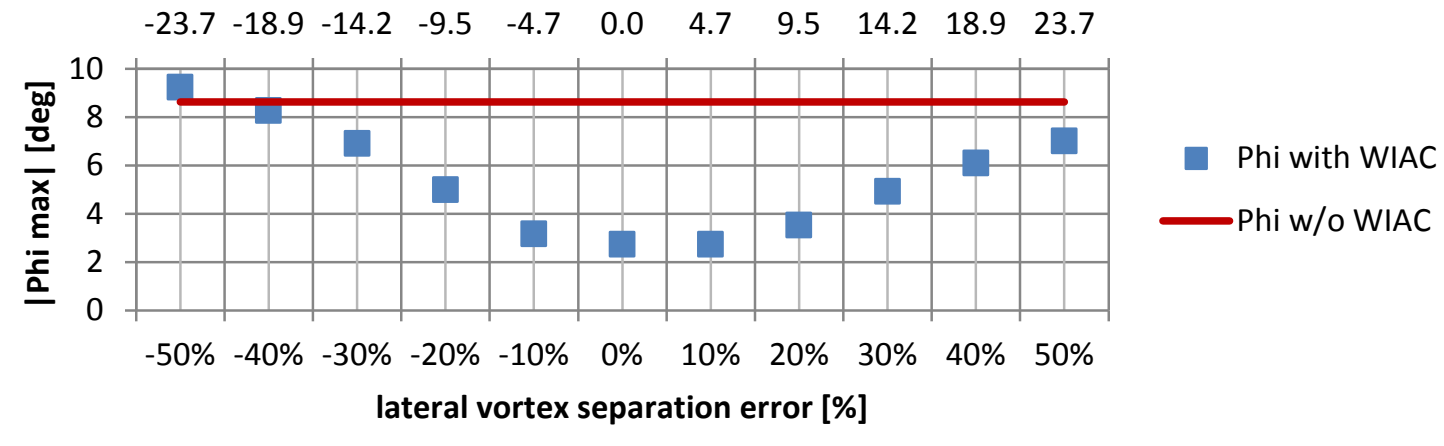

Figure 13. Impairment of reduction of maximum bank angle amplitude by the wake impact alleviation controller due to error of identified lateral vortex separation.

An unexpected observation concerning the influence of the error of the identified wake vortex circulation in Fig. 12 is the fact that the minimum bank angle does not occur in the case with the correctly identified vortex strength but for a $20 \%$ larger value. The reason for this effect can be attributed to the interaction between the basic control system and the wake impact alleviation control function. In the case of no identification error the time history of the Euler angles corresponds to Fig. 6. The remaining peak of the bank angle at $8.5 \mathrm{~s}$ when the wake impact alleviation controller is applied results from the fact that the basic control system commands an aileron deflection opposing the WIAC aileron command. This aileron command originates from the lateral control design which includes a decoupling of the roll and yaw axis responses to external disturbances. As a consequence the WIAC command that would be necessary to compensate for the wake-induced roll reaction is not fully reached and a small bank angle remains. If the identified wake vortex circulation is, however, larger than the actual vortex strength, this leads to an overestimation of the wake-induced rolling moment by the wake impact alleviation control function. It thus commands larger aileron deflections than would actually be needed to countervail the disturbance. This overestimated command compensates for the unfavorable influence of the opposing aileron deflection command by the basic control system and the resulting aileron deflection corresponds to the required position to suppress the wake-induced roll response. This explains why small deviations from the true wake vortex parameters 
can result in a lower maximum bank angles if the wake impact alleviation system is applied in combination with a basic control system.
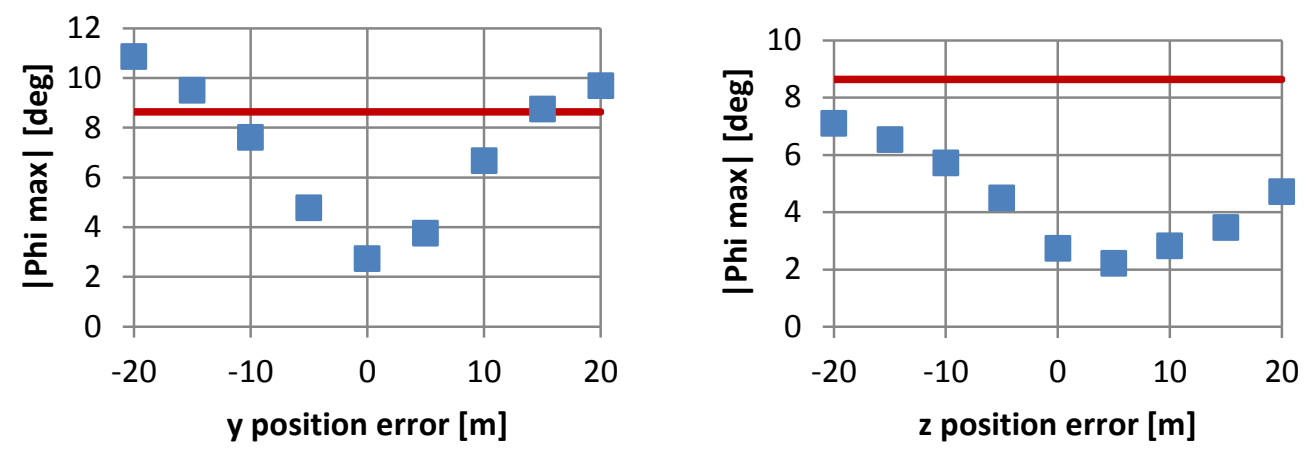

\section{Figure 14. Impairment of reduction of maximum bank angle amplitude by the wake impact alleviation controller due to error of position of wake vortex centerline.}

Figure 14 shows the influence of errors in the identified position of the wake vortex centerline. It can be seen that the lateral y position error is a very critical parameter for a successful application of the WIAC. The restrictions for the lateral position error are very tight and errors greater than $+/-10 \mathrm{~m}$ will lead to an increase in the maximum occurring bank angle compared to the case without WIAC deployed. The error of the identified lateral position means that both vortices are shifted parallelly which corresponds to a pure temporal shift of the detected disturbance moments in relation to the actual wake-induced moments. ${ }^{\S}$ The applied additional control surface deflections thus easily exhibit incorrect signs and unfavorably support the disturbance. The effect that the wake vortex is shifted in the horizontal plane is the same for an error of the lateral position of the vortex centerline and an incorrectly identified lateral vortex separation respectively. Nevertheless, a lateral position error leads to a bigger impairment of the attainable bank angle alleviation than an error of the vortex separation of the same magnitude. The incorrect lateral separation is distributed to two vortex cores, whereupon the y position error affects both vortex cores. For a $10 \mathrm{~m}$ error of the vortex separation each core has a position error of $5 \mathrm{~m}$. A $10 \mathrm{~m}$ error of the vortex centerline means that both cores are shifted by $10 \mathrm{~m}$. Additionally, the wake-induced disturbance velocity at any particular location is composed of the sum of the induced velocities of both vortex cores. In the case of a y position error, the sum of the induced velocities of both vortex cores stays the same at the same relative positions with respect to the location of the vortex centerline. If the lateral separation of the cores varies, the sum of the wake-induced velocities at each position varies as well. This affects the shape of the disturbance, whereas the lateral error leads to a temporal shift and consequently more easily to aggravating control commands.

The comparison of the effects of position errors in the $y$ and the $\mathrm{z}$ axis shows that the success of the wake impact alleviation is less sensitive to an error in the vertical position than to an incorrect detection of the lateral position. In contrast to lateral position errors, inaccurately identified vertical positions do not shift the disturbance moments in time but modify the shape of the induced moments. The moment that is primarily affected by a faultily detected vertical position is the yawing moment. If the vertical position of the wake vortex is detected on the other side of the aircraft the lateral wake-induced wind velocities change sign and result in an inverted yawing moment. For the vertical wind velocities a change of the vertical wake vortex position only modifies the amplitude but not the shape of the wind velocities. The detected rolling moment, which mainly results from the vertical wake vortex wind velocities, consequently only varies in magnitude for inaccurately identified vertical positions of the wake vortex but does not change its shape. This explains why the impairment of the bank angle reduction is not as strong for an incorrect vertical wake vortex position as for an error of the identified lateral position. However, it must be noted that for a different encounter scenario where the true wake vortex has a large vertical offset to the aircraft, a too

\footnotetext{
$\S$ For the considered $15^{\circ}$ lateral encounter scenario with an airspeed of $150 \mathrm{kt}$ a lateral position error of $10 \mathrm{~m}$ corresponds to a temporal shift of the disturbance moments of $0.5 \mathrm{~s}$.
} 
closely detected vertical vortex position can lead to an overcompensation and thus an increase of the bank angle compared to the case without the wake impact alleviation control.

The shift of the minimum bank angle from the actual vortex position to an vertical position error of $5 \mathrm{~m}$ has the same reason as explained for the circulation error. At the moment when largest bank angle occurs during the encounter, the aircraft sinks a few meters and is located closer to the inaccurately detected wake vortex position than to the actual vortex cores. This leads to an overestimation of the aileron deflection by the WIAC and thus a compensation of the unfavorable influence of the opposing roll command of the basic control system.
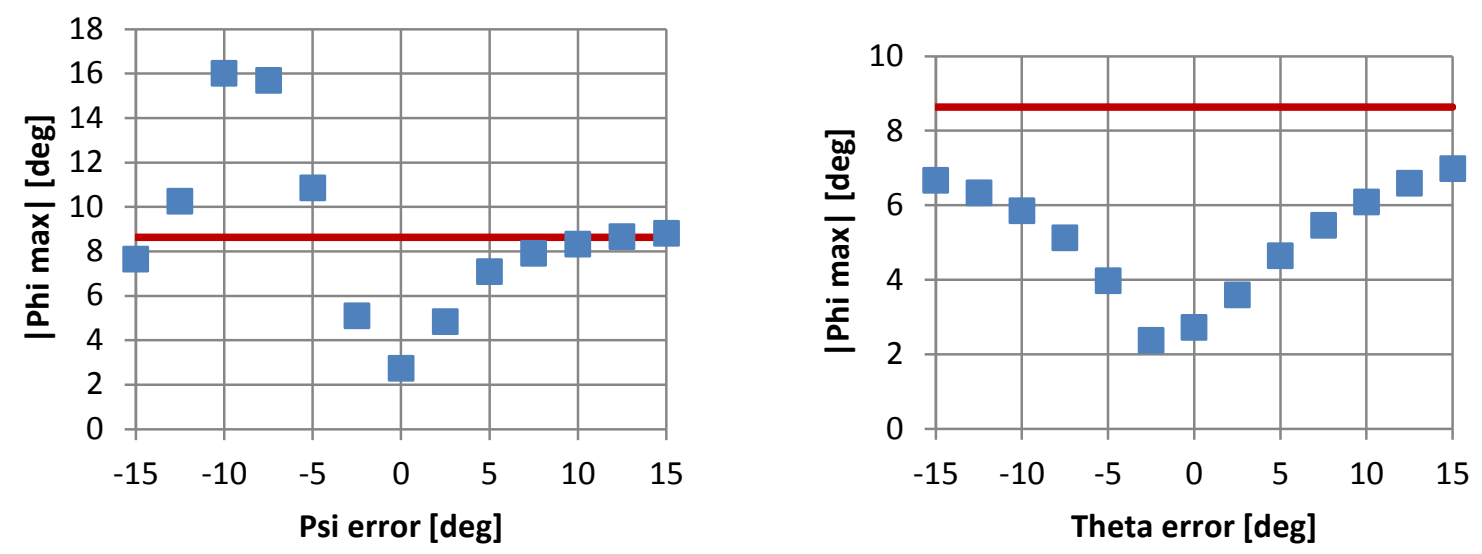

Figure 15. Impairment of reduction of maximum bank angle amplitude by the wake impact alleviation controller due to error of wake vortex orientation.

The variation of the error of the lateral and vertical encounter angle, illustrated in Fig. 15, is performed in such a way that only the encounter angle is changed. The position of the vortex centerline is adapted such that the intersection of the trimmed flight path and the vortex centerline is always at the same distance in the flight direction with respect to the point where the wake vortex has been detected. The variation of the wake elevation has similar effects to a change in the vertical position of the vortex centerline because it influences the vertical distance of the wake vortex relative to the aircraft position. The best bank angle reduction occurs for an elevation error of $-2.5^{\circ}$; the overestimated wake-induced rolling moment again leads to an compensation of the on the counteracting aileron command of the basic control system. Altogether the wake impact alleviation is comparably robust against errors of the identified wake elevation. Even at $+/-15^{\circ}$ the maximum bank angle can still be reduced.

Similarly to the lateral position error, an error of the identified wake azimuth also has much greater consequences than the variation of the wake elevation. Very small deviations, such as $-5^{\circ}$, result in the wake impact alleviation control function aggravating the bank angle response of the aircraft. Therefore, it is thus very important that this parameter is identified with an appropriate precision. For negative wake azimuth errors the impairment of the bank angle reduction is much stronger than for positive errors. This results from the fact that for a larger azimuth the encounter angle increases and the rate of change of the detected disturbance moments increases. The control surface deflections commanded by the wake impact alleviation control function are thus so fast that the aircraft response is hardly effected. In the case of negative errors of the wake azimuth the assumed encounter angle becomes smaller and the wake impact alleviation control function commands incorrect control surface deflections over a long period of time resulting in aggravated roll responses of the aircraft. In the particular case of a $-15^{\circ}$ error the identified wake vortex is located parallelly, exactly above the trimmed flight path of the aircraft. The detected wakeinduced rolling moment is thus almost zero because the wind velocities of the left and right vortex core compensate each other and the WIAC roll control commands are very small.

In the presented analysis only separate variations of the wake vortex parameter estimation errors were considered. Obviously estimations errors can occur simultaneously and the resulting effects cannot be directly predicted from the separate variation analysis. Moreover, the specific values at which the benefit of the wake impact alleviation control system is eliminated varies according to the encounter geometry. Nevertheless, the analysis 
presented here gives an impression of the different sensitivities for the wake impact alleviation with respect to the errors of the identified wake vortex parameters. A successful application of the wake impact alleviation controller requires that the wake vortex is identified with an accuracy that stays within the determined tolerances of the wake vortex parameters.

\section{Conclusions and Outlook}

The work presented outlines a possible realization of a wake impact alleviation control system on the basis of a forward-looking LiDAR sensor. The new approach of the concept is the combination of the wake impact alleviation with a wake identification algorithm which allows the application of the alleviation control system with sensors providing line-of-sight measurements only. As LiDAR sensors will most likely only be realizable as LoS sensors in the near future, this aspect is very important for a feasible implementation of the system. The wake identification algorithm is used to identify the characteristic parameters of a wake vortex model describing the disturbance. The wake impact alleviation system uses this identified wake vortex model to determine the wake-induced disturbance moments and commands additional control surface deflections to compensate for these moments. It was demonstrated that this concept works well in order to alleviate the wake-induced moments under idealized conditions assuming that the wake vortex parameters are perfectly identified. However, the analysis also shows that the sole alleviation of the pitching moment leads to increased flight path deviation in the vertical direction, which might be critical during approach and landing. This shows that the optimal alleviation strategy in the longitudinal axis still need to be examined. The desired aircraft dynamics during the wake encounter need to be analyzed in detail. Following this analysis the specific goal of the wake impact alleviation can then be defined. The result might lead to the alleviation of a combined factor of the wake-induced pitch moment and vertical force.

The application of the wake impact alleviation controller in combination with the wake identification demonstrated that the concept is also successful in reducing the wake-induced attitude change for the most critical lateral encounter angles if the wake parameters describing the disturbance originate from the actual identification. Only for steep encounters of $35^{\circ}$ the wake identification based alleviation was no longer successful. Nevertheless, during this encounter the aircraft response was very small and the encounter could be classified as uncritical.

A sensitivity study of the identified wake vortex parameters showed that the success of the wake impact alleviation is most sensitive to the wake azimuth and lateral position of the vortex centerline relative to the aircraft, followed by the separation of the vortex cores. If the errors of these parameters get too large, the application of the WIAC can lead to an aggravation of the wake-induced bank angle response. The vortex circulation has been found to be the least sensitive parameter for the bank angle alleviation. Even for very large errors of the identified vortex circulation the attainable reduction of the maximum bank angle is only impaired but does not lead to an increased response.

It has to be noticed that the present analysis is performed under some simplified conditions. The wake identification is performed in a simplified simulation in which the aircraft is not influenced by the wake vortex. Once the OWI has converged to a valid result, the identified wake vortex parameters are used for the wake impact alleviation. This approach is simplified in the sense that the measurement directions of the LiDAR sensor do not change their direction because the aircraft attitude stays constant. If the aircraft attitude was already influenced by the wake vortex the scan angles might have to be increased in order to get enough information about the wake vortex. As the wake identification provides a valid result far enough in advance before the aircraft reaches the vortex core, the assumption that the aircraft is not affected yet by the wake vortex appears justified. Nonetheless, more detailed analyses will be performed concerning the effect of the wake-induced aircraft motion on the wake identification during future work. Another simplified approach is the application of the same aerodynamic interaction model for the simulation environment and the wake impact alleviation controller. So far the aerodynamic interaction model used in the wake impact alleviation control system to derive the wake-induced moments is the same as the AIM applied in the simulation to model the interaction of the wake vortex with the aircraft. If the wake vortex is perfectly identified, the wake impact alleviation hence determines the exact disturbance acting on the aircraft in the simulation environment used here. Under real flight conditions the AIM in the wake impact alleviation

control system will not be able to predict the wake-induced aircraft response so perfectly. Furthermore, the 
simulation results presented in this paper use a simplified analytical wake vortex model. Realistic wake vortices are much more complex than the model applied here, and deviate from the shape of straight lines of the analytical models due to the vortex decay process. Finally, it has to be stated that a model of a LiDAR sensor was used in the simulations which itself contains errors with respect to a possible real LiDAR sensor. The influence of these simplifications needs to be assessed in detail in future analyses. Future work will include a detailed assessment of the success of the wake impact alleviation function for more realistically modeled wake vortices. For this purpose wake vortices determined by Large Eddy Simulation (LES) will be integrated into the simulation and the wake vortices of different ages including deformed vortices will also be considered. In addition, the robustness of the wake impact alleviation control system with respect to modeling errors of the aerodynamic interaction model will be assessed. Another issue for future research would be an improved integration of the WIAC and the existing aircraft flight control system to prevent undesired interaction of the basic control system during a wake vortex encounter. Despite a lot of influences on the wake impact alleviation control system still need to be examined, the present analysis demonstrates that the alleviation system shows good results for the considered conditions and that the concept is very promising.

\section{Acknowledgments}

Parts of the presented results have been achieved during a sub-contract funded by AIRBUS Operations SAS. The authors would like to thank Airbus and all colleagues at DLR who contributed to this work for their support.

\section{References}

${ }^{1}$ National Transportation Safety Board, "In-Flight Separation of Vertical Stabilizer American Airlines Flight 587 Airbus Industrie A300-605R, N14053 Belle Harbor, New York November 12, 2001”, Aircraft Accident Report NTSB/AAR-04/04, Washington, D.C., 2004.

${ }^{2}$ National Transportation Safety Board, Brief of Accident LAX94FA073, Washington, D.C. October 06, 1994.

${ }^{3}$ Fock, H., Hahn, K.-U., Schwarz, C., "Verwendung von vorausschauenden Strömungssensoren in Pilotenassistenzsystemen für den Wirbelschleppeneinflug (English: Application of Forward-Looking Flow Sensors for Pilot Assistance Systems for Wake Vortex Encounters),” DLR Report IB 111-2006/12, German Aerospace Center DLR, Braunschweig, Germany, March 2006.

${ }^{4}$ Schwarz, C., Hahn, K.-U., “Automated Pilot Assistance for Wake Vortex Encounters”, CEAS, Berlin, Germany, 10 - 13 September 2006.

${ }^{5}$ Hahn, K.-U., Fischenberg, D., Niedermeier, D., Horn, C., "Wake Encounter Flight Control Assistance Based on ForwardLooking Measurement Processing”, AIAA Guidance, Navigation, and Control Conference, Toronto, Ontario Canada, 2 - 5 August 2010.

${ }^{6}$ Kloidt, S., „Beiträge zum Entwurf eines Flugregelungssystems zur Reduktion des Wirbelschleppeneinflusses (English: Contributions to the Design of a Flight Control System for the Reduction of the Wake Vortex Impact)“, Ph.D. Dissertation, TU Berlin, Germany, 2007.

${ }^{7}$ Looye, G., Lombaerts, T., Kier, T., “Design and Flight Testing of Feedback Control Laws”, Research Report 2012-02, The DLR Project Wetter \& Fliegen, p. 162-170, German Aerospace Center, Köln, February 2012.

${ }^{8}$ Rafi, M., Steck, J., "Reponse and Recovery of an MRAC Advanced Flight Control System to Wake Vortex Encounters", AIAA Infotech@Aerospace Conference, Boston, August 2013.

${ }^{9}$ Fischenberg, D., “Online Wake Identification Algorithms Using Forward Looking LIDAR Sensor Measurements”, DLR Report IB111-2013/11, German Aerospace Center DLR, Braunschweig, Germany, Feburary 2006.

${ }^{10}$ Fischenberg, D., "Strömungsermittlungsverfahren/Flow Determination Method/Procédé de détermination d‘écoulement”, Patent No. EP 2340438 B1, European Patent Office 2013.

${ }^{11}$ Burnham, D. C., Hallock, J. N., “Chicago Monoacoustic Vortex Sensing System”, Vol. 4, Wale Vortex Decay, Springfield, VA, National Information Service, 1982.

${ }^{12}$ Fischenberg, D., “A method to validate wake vortex encounter models from flight test data”, ICAS 2010, 27th International Congress of the Aeronautical Sciences, Nice, France, 2010.

${ }^{13}$ Farineau, J., "Lateral Electric Flight Control Laws of A Civil Aircraft Based Upon Eigen Structure Assignment Technique”, AIAA Guidance, Navigation, and Control Conference, Boston, USA, August 1998.

${ }^{14}$ Jategaonkar, R. V., "Flight Vehicle System Identification: A Time Domain Methodology ”, Progress in Astronautics and Aeronautics, AIAA, Reston, VA, 2006, pp. 86-87. 